\title{
Coordinated Control of a Dual-Stage Positioning System Using Constrained Model Predictive Control
}

\author{
Haghighat, S.; Di Cairano, S.; Konobrytskyi, D.; Bortoff, S.A.
}

TR2014-099 October 2014

\begin{abstract}
Dual-stage positioning systems have been widely used in factory automation, robotic manipulators, high-density data storage systems, and manufacturing systems. Trajectory generation and control of dual-stage positioning systems is of great importance and is made complicated by the presence of physical and operational constraints. In this work, we describe how to generate feasible reference trajectories for a dual-stage positioning system consisting of a fine stage and a coarse stage, and how to use them in a model predictive control algorithm for which recursive feasibility is guaranteed. The reference generation algorithm is guaranteed to generate trajectories that satisfy all the constraints for the fine and coarse stages. We also describe a constrained model predictive control algorithm used to control the coarse stage. The simulation results of applying the developed methodology to track a pre-determined pattern is presented.
\end{abstract}

ASME Proceedings - Advances in Motion Control

This work may not be copied or reproduced in whole or in part for any commercial purpose. Permission to copy in whole or in part without payment of fee is granted for nonprofit educational and research purposes provided that all such whole or partial copies include the following: a notice that such copying is by permission of Mitsubishi Electric Research Laboratories, Inc.; an acknowledgment of the authors and individual contributions to the work; and all applicable portions of the copyright notice. Copying, reproduction, or republishing for any other purpose shall require a license with payment of fee to Mitsubishi Electric Research Laboratories, Inc. All rights reserved. 



\section{COORDINATED CONTROL OF A DUAL-STAGE POSITIONING SYSTEM USING CONSTRAINED MODEL PREDICTIVE CONTROL}

\author{
Sohrab Haghighat \\ Mitsubishi Electric Research Laboratories \\ Cambridge, MA 02139 \\ Email: haghighat@merl.com
}

\author{
Dmytro Konobrytskyi \\ Mitsubishi Electric Research Laboratories \\ Cambridge, MA 02139 \\ Email: konobrytskyi@merl.com
}

\author{
Stefano Di Cairano \\ Mitsubishi Electric Research Laboratories \\ Cambridge, MA 02139 \\ Email: dicairano@merl.com \\ Scott Bortoff \\ Mitsubishi Electric Research Laboratories \\ Cambridge, MA 02139 \\ Email: bortoff@merl.com
}

\begin{abstract}
Dual-stage positioning systems have been widely used in factory automation, robotic manipulators, high-density data storage systems, and manufacturing systems. Trajectory generation and control of dual-stage positioning systems is of great importance and is made complicated by the presence of physical and operational constraints. In this work, we describe how to generate feasible reference trajectories for a dual-stage positioning system consisting of a fine stage and a coarse stage, and how to use them in a model predictive control algorithm for which recursive feasibility is guaranteed. The reference generation algorithm is guaranteed to generate trajectories that satisfy all the constraints for the fine and coarse stages. We also describe a constrained model predictive control algorithm used to control the coarse stage. The results of applying the developed methodology to track a pre-determined pattern is presented.
\end{abstract}

\section{INTRODUCTION}

Dual-stage actuation has been used in different positioning systems such as robotic manipulators [1], xy-stage positioners [2-4], and optical/hard disk drives [5-7]. The main motivation behind the use of double-stage mechanisms is their capability to overcome the limitations of single-stage actuation sys- tems such as limited bandwidth or small actuation range, the so called 'stroke'. Conventional positioning systems such as electrical motors or hydraulic actuators are generally bounded by their low speed and limited bandwidth. On the contrary, the more recently developed fine positioners such as piezoelectric actuators, and inertia-less light deflectors can be driven at much higher velocities and accelerations but are limited by their small range of motion. Using dual-stage actuation, one can design a system that has an overall bandwidth of the fine stage while leveraging the larger range of motion of the coarse stage [2].

When generating a reference trajectory for a dual-stage positioning system, numerous constraints have to be considered due to physical limitations (actuator strokes) and operational limits (maximal velocities and accelerations). Particularly, the fine stage stroke constraint requires the difference between the target position and the coarse stage position to be within the range of the fine stage.

To satisfy fine stage stroke constraints, Swon and Cheong [4] took advantage of the extra degree of freedom, by using a nullmotion control approach, and minimized the deviation of the fine stage from its neutral position. This approach, however, does not offer any guarantees for the satisfaction of the fine stage stroke constraint. Michellod et al. [8] addressed the fine stage stroke saturation by heuristically designing a partial observer and clos- 
ing the coarse stage control loop using the coarse stage position, while closing the fine stage control loop using the overall system position. This approach forces the coarse stage to continue towards the reference signal even when the combined position matches the desired position. As a result, the fine positioner has the tendency to return to its neutral position.

Following the $P Q$ design method laid out by Schroeck et al. [5], Ryoo et al. proposed a design technique to guarantee the satisfaction of the fine actuator stroke constraints used in an optical disk drive control [6]. The reference trajectory was constructed as the combination of a slowly increasing ramp signal and small oscillations of the same frequency as the disk rotational frequency. While this method can guarantee constraint satisfaction, it requires the prior knowledge of the ramp slope.

Recently, Lam et al. have used MPC for contouring control in a single-stage $\mathrm{X}-\mathrm{Y}$ table [9]. A multi-objective cost function that reflects a tradeoff between the contouring accuracy and the path speed is optimized, subject to state and input constraints. However, as a single-stage actuator is used, the stroke constraints were not considered.

To the best of the authors' knowledge, none of the existing approaches in the literature can offer guaranteed constraint satisfaction in a dual-stage mechanism while tracking a generic reference trajectory. In this paper, we use model predictive control (MPC) to steer the coarse stage. To guarantee recursive feasibility, we develop a governor to generate reference trajectories for the coarse and fine stages of the machine. We prove that, by using the generated reference trajectory for the coarse stage as reference for the MPC, we obtain recursive feasibility of the MPC problem, since the reference is feasible. Exploiting the feasible trajectory generated by the governor, the MPC finds the constrained optimal trajectory. This paper is organized as follows. Section 2 introduces the coarse and fine stage dynamics and their constraints. It also provides a formal description of the problem. Section 3 focuses on the development of the governor and reference assignment to the coarse and fine stages. Section 4 describes the MPC design for the coarse stage. Finally, Section 5 shows, through numerical simulation, the capability of the developed control architecture in controlling a dual-stage xy-positioning system, which is used to inspect a list of target points on a workpiece.

Notation: $\mathbb{R}$ is the set of real numbers and $\mathbb{Z}_{+}$is the set of positive integer numbers. For a discrete-time signal $x \in \mathbb{R}^{n}$ with sampling period $T, x(k)$ is the state at sampling instant $k$, i.e., at time $T k$. By $[x]_{i}$ we denote the $i$-th component of vector $x$. Vector inequalities are considered component-wise. With a little abuse of notation $\|x\|_{Q}^{2}=x^{\prime} Q x$.

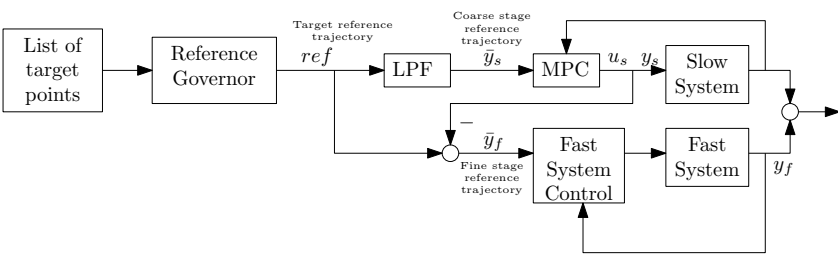

Figure 1. The control structure of a dual-stage positioning system

\section{CONTROL PROBLEM, ASSUMPTIONS, AND CON- STRAINTS}

In this work, a dual-stage positioning system is used to inspect a list of pre-determined target points on a workpiece. The system is composed of an XY-table, i.e., a table with actuators that allow it to move in the $\mathrm{X}$ and $\mathrm{Y}$ directions, referred to as the coarse stage, and an optical beam steering system, referred to as the fine stage. The optical system is not mounted on the XYtable and therefore, the two stages are not dynamically coupled, however, as the XY-table moves, the position of the target point with respect to the optical system is changed. The control system architecture for the overall system is shown in Fig. 1. The governor takes a list of target points $P(i)$ (pairs of $\mathrm{x}$ and y positions) and generates a continuous, feasible (timed) reference trajectory $(r e f)$. A filtered reference trajectory, $\bar{y}_{s}$, that contains the low frequency content of the reference trajectory ( $r e f$ ) is given as target to an MPC controller. The MPC controller actuates the coarse stage and generates a trajectory, $y_{s}$, that tracks target $\bar{y}_{s}$ such that ref is always within the range of motion of the fine stage. The fine stage controller tracks the difference between the target reference trajectory, $r e f$, and the coarse stage trajectory $y_{s}$

$$
\bar{y}_{f}=\operatorname{ref}-y_{s},
$$

so that all the target positions are achieved by the steered beam. This is possible because the fine stage is several orders of magnitude faster than the coarse stage and our proposed control algorithm guarantees that the magnitude of $\bar{y}_{f}$ is always less than the stroke of the fine stage. Given the significant difference between the bandwidth of the fine and coarse stages, the target reference trajectory $(r e f)$ and the fine stage reference trajectory $\left(\bar{y}_{f}\right)$ are generated with a significantly shorter sampling period $\left(T_{f}\right)$ than the coarse stage reference trajectory $\left(\bar{y}_{s}\right)$ which is discretized using the sampling period $T_{s}$.

$$
T_{f}=\frac{T_{s}}{N_{f}}, N_{f} \in \mathbb{Z}_{0}
$$

where $N_{f}$ is the ratio of the coarse stage sampling period to the fine stage sampling period. Position measurements for the coarse 
and fine stages are available for feedback. For the system considered here, the $\mathrm{X}$ and $\mathrm{Y}$ axes of the coarse and fine stages are decoupled and each axis is controlled independently. Hence, from now on we will present the equations related to one axis only while it is intended that two equal sets of equations (possibly with different parameter values) are implemented. The equation of motion for the coarse stage along each axis is

$$
J_{s} \ddot{y}_{s}+b_{s} \dot{y}_{s}=L u_{s}
$$

where $y_{s}$ is the linear displacement of the coarse stage, $J_{s}$ is the lumped inertia, $b_{s}$ is the lumped damping, and $u_{s}$ is the applied torque. The above equation is time-discretized with sampling period $T_{S}$ and is transformed into state-space form. For each axis, the discrete-time dynamic equation is of the form

$$
\begin{gathered}
x_{s}(k+1)=A x_{s}(k)+B u_{s}(k) \\
y_{s}(k)=C x_{s}(k),
\end{gathered}
$$

where $x_{s}(k) \in \mathbb{R}^{2}$ is a vector representing the position and velocity of each axis, $u_{s}(k) \in \mathbb{R}$ is the input torque for each axis, and $y_{s}(k) \in \mathbb{R}$ is the displacement of each axis.

The target dual-stage positioning system has a number of operational and physical constraints that must be considered in the design of the control system. The coarse stage has stroke and speed constraints along both axes. Furthermore, a limited torque can only be provided by the XY-Table servomotors.

$$
\begin{aligned}
\left|x_{s}(k)\right| \leq & {\left[\begin{array}{l}
d_{\max , s} \\
v_{\max , s}
\end{array}\right] } \\
\left|u_{s}(k)\right| & \leq u_{\max , s},
\end{aligned}
$$

where $d_{\max , s}$ denotes the stroke constraint, $v_{\max , s}$ is the velocity constraint, and $u_{\max , s}$ represents the maximum torque. The fine stage can be represented by $2^{\text {nd }}$ order dynamics as

$$
Y_{f}(s)=\frac{\omega_{f}^{2}}{s^{2}+2 \zeta_{f} \omega_{f} s+\omega_{f}^{2}} U_{f}(s),
$$

where $y_{f}$ is the fine stage position. The fine stage has stroke $d_{\max , f}$, velocity $v_{\max , f}$, and acceleration $a_{\max , f}$ constraints.

Following the above description, the problem of controlling a dual-stage positioning systems can be divided into two subproblems.

Problem 1 (Reference generation). Given the coarse stage dynamics (4), and constraints (5), generate in receding horizon a target reference trajectory, ref, and a filtered reference trajectory, $\bar{y}_{s}$, such that (1) ref connects a series of predefined target points, (2) $\bar{y}_{s}$ can be continuously tracked by the coarse stage while the tracking error is smaller than $d_{\max , f}$, and (3) $\bar{y}_{f}=r e f-y_{s}$ can be tracked by the fine stage without violating its velocity and acceleration constraints.

Problem 2 (Coarse stage control). Using the reference trajectory from Problem 1, design a control system for the coarse stage to continuously track $\bar{y}_{s}$, while keeping the tracking error smaller than $d_{\max , f}$, and enforcing all the physical and operational constraints of the coarse stage.

In fact, a third problem should be considered, that is, to control the fine stage such that the overall position of the system is equal to the target position. However, if Problem 1 and Problem 2 are correctly solved, this additional problem can be addressed using standard control techniques, e.g. PID and is not further discussed in this work.

As mentioned before, we use MPC to control the coarse stage. The following general MPC formulation can be used to control the coarse stage.

$$
\begin{aligned}
\min _{U(k)} & \sum_{i=1}^{N}\left\|\bar{y}_{s}(k+i)-y_{s}(k+i)\right\|_{2}^{Q}+\left\|u_{s}(k+i-1)\right\|_{2}^{R} \\
\text { s.t. } & x_{S}(k+1)=A x_{S}(k)+B u_{s}(k) \\
& y_{s}(k)=C x_{s}(k) \\
& \left|u_{s}(k+i)\right| \leq u_{\max , s}, i=0, \cdots, N-1 \\
& \left|x_{s}(k+i)\right| \leq\left[\begin{array}{c}
d_{\max , s} \\
v_{\max , s}
\end{array}\right] \quad i=1, \cdots, N \\
& x_{S}(k+N)=\bar{x}_{s}(k+N) .
\end{aligned}
$$

However, this MPC problem (7) lacks the appropriate constraints required for the satisfaction of the fine stage stroke constraint $\left(\left|\bar{y}_{f}\right| \leq d_{\max , f}\right)$. Furthermore, as a tracking problem is solved in this work, a modified terminal constraint is used (7f). The standard terminal constraint which is used in regulating problems, enforces the states at the end of the horizon to be at the origin. Here, we are tracking a continuously varying trajectory, and therefore, the states at the end of the horizon should be equal to the reference value. In addition to modifying the terminal constraint (as in 7f), to guarantee recursive feasibility the coarse stage reference trajectory, $\bar{y}_{s}$, has to satisfy some requirements. Note that without guaranteeing the recursive feasibility of the MPC, the satisfaction of the machine constraints cannot be guaranteed. In the following two sections, we will formulate the required conditions for the coarse stage reference trajectory and develop the additional constraints needed for the MPC problem. Furthermore, we will design an algorithm for generating reference trajectories that satisfy such conditions, thus ensuring that a solution for the MPC problem always exists. 

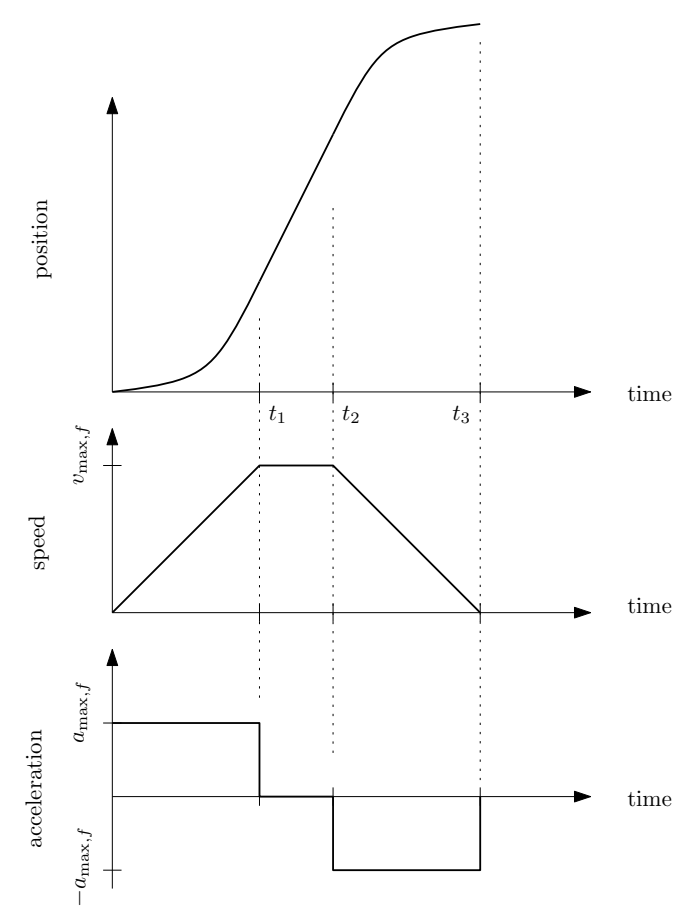

Figure 2. Beam spot reference trajectory profile between two holes

\section{REFERENCE GENERATION}

In order to solve Problem 1, we first construct a target reference trajectory, $r e f$, based on the fine stage acceleration and velocity constraints. The reference trajectory is generated by joining together a sequence of sub-trajectories, each connecting consecutive target points. A bang-bang acceleration profile is used to generate the sub-trajectories while satisfying the constraints. During the acceleration (deceleration) phases of the subtrajectory, the fine stage maximal acceleration (deceleration) is applied. When the maximal speed is reached, the acceleration is set to zero. Hara et al. [10] have adopted a similar approach to generate reference trajectories for hard disk drives. The acceleration, velocity, and position profiles of the sub-trajectory between two consecutive target points are shown in Fig. 2.

Depending on the distance between the two target points, the time indices $t_{1}$ to $t_{3}$ can be found as follows

$$
\begin{cases}t_{1}=t_{2}=\sqrt{\frac{d}{a_{\max }}} & d \leq \frac{v_{\max }^{2}}{a_{\max }} \\ t_{3}=2 \sqrt{\frac{d}{a_{\max }}} & d>\frac{v_{\max }^{2}}{a_{\max }} \\ t_{1}=\frac{v_{\max }}{a_{\max }} & \\ t_{2}=t_{1}+\left(\frac{d}{v_{\max }}-\frac{v_{\max }}{a_{\max }}\right) & \\ t_{3}=t_{2}+t_{1} & \end{cases}
$$

where $d$ is the distance between two consecutive points and $\frac{v_{\max }^{2}}{a_{\max }}$ is the shortest distance at which the maximum speed is reached. Note that, although, the $\mathrm{X}$ and $\mathrm{Y}$ axes are not dynamically coupled, the fact that both coordinates must be at the target point at the same time requires some coordination between the axes. As a result, the distance, $d$, used to compute $t_{i}, i=\{1,2,3\}$ in (8) is

$$
d=\max \left\{\left|[P(i+1)]_{1}-[P(i)]_{1}\right|,\left|[P(i+1)]_{2}-[P(i)]_{2}\right|\right\}
$$

where $P(i), P(i+1)$ are the two consecutive target points the sub-trajectory is joining.

Since the target reference trajectory is generated using the fine stage operational constraints, if the target reference trajectory is within the reach of the fine stage, it can be feasibly tracked. Therefore, we have to control the coarse stage to capture the main movement of the target reference trajectory, ref, such that the target is always within the stroke of the fine stage. To this end, a low-pass filter is used to extract the low-frequency content of the target reference trajectory. In this work, a non-causal finite impulse response (FIR) filter has been employed to smoothen the target reference trajectory. As the reference trajectory for the coarse stage $\left(\bar{y}_{s}\right)$ is used as target by the MPC algorithm, due to the predictive nature of MPC, the coarse stage trajectory has to be computed for $N$ steps (control horizon) in the future. Therefore, the coarse stage reference trajectory at time $k+N$ has to be computed at the time step $k$.

$$
\begin{aligned}
& \bar{y}_{s}(k+N)=\sum_{n=-N_{\mathrm{fir}}}^{N_{\mathrm{fir}}} h(n) \operatorname{ref}(k+N-n) \\
& \text { where } h(n)=\frac{4 N_{\mathrm{fir}}}{\pi} \cos \left(\frac{n \pi}{2 N_{\mathrm{fir}}}\right), n=-N_{\mathrm{fir}}, \cdots, N_{\mathrm{fir}}
\end{aligned}
$$

In the above equation, $N_{\text {fir }} \in \mathbb{Z}_{+}$is the filter half window. The non-causal FIR filter is centered around the time step $k+N$. This is illustrated in Fig. 3. As shown by Schroeck et al. [5] significant phase difference between the coarse and fine reference trajectories, especially at mid-frequencies results in destructive interference between the two stages. The application of a noncausal filter avoids the introduction of any phase lag into the coarse stage reference trajectory, but requires the computation of the target reference trajectory, re $f$, to be evaluated for $N_{\mathrm{fir}} T_{f}$ seconds ahead of the coarse stage reference trajectory. Thus, for real-time application at least part of the (spatial) trajectory needs to be known in advance, which is consistent with the target applications in manufacturing and with the use of MPC proposed here.

\subsection{TRAJECTORY FEASIBILITY VERIFICATION}

According to the operation of MPC, which uses the reference, the reference is generated in a receding horizon fashion. 


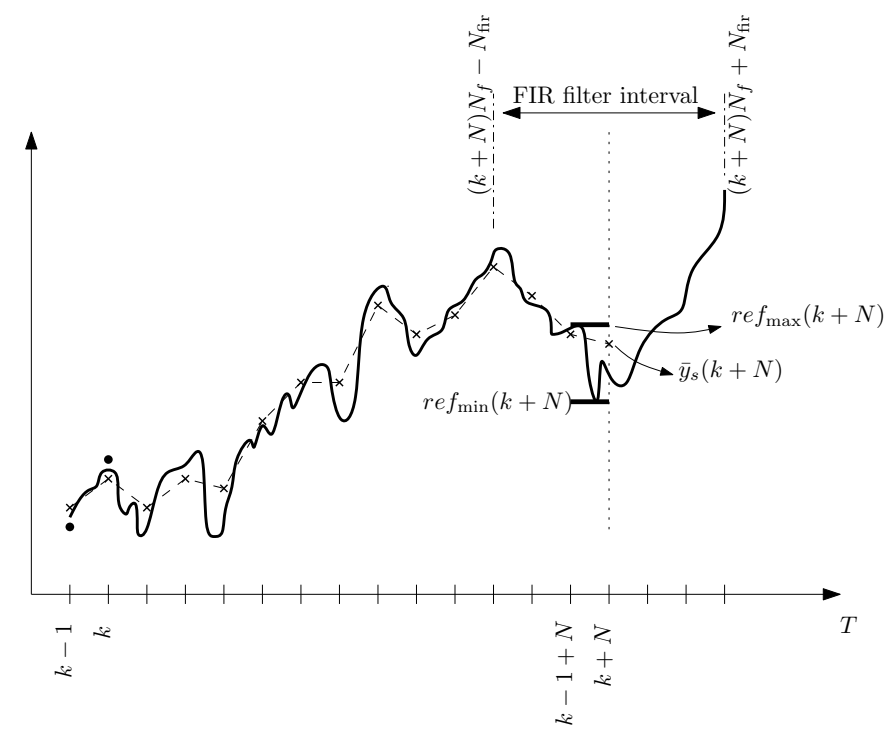

Figure 3. FIR filter is used to generated a new reference point for the coarse stage.

Thus, starting from a feasible reference portion, we extend the coarse stage reference, $\bar{y}_{s}$, in a way that preserves feasibility. This means that the feasibility has to be maintained as a new reference point is added to the coarse stage reference trajectory. For this application, the feasibility of the coarse stage reference trajectory, $\bar{y}_{S}$ can be verified by checking two constraints: (1) recursive feasibility constraint and (2) inter-sample tracking constraint.

3.1.1 RECURSIVE FEASIBILITY As indicated in (7), we use a terminal constraint to guarantee recursive feasibility of the MPC problem [11]. In addition to a terminal constraint, to maintain the feasibility of a tracking MPC problem, the reference trajectory has to satisfy the following forward reachability condition.

$$
\bar{x}_{s}(k+N) \in \operatorname{Reach}\left(\bar{x}_{s}(k+N-1)\right)
$$

where $\bar{x}_{s}$ is the reference state for the coarse stage and Reach is defined as

$$
\begin{array}{r}
\operatorname{Reach}(X)=\left\{x^{+} \in \mathbb{R}^{n}: \exists x \in X, \exists u \in \mathcal{U}\right. \\
\text { s.t. } \left.x^{+}=A x+B u\right\}
\end{array}
$$

To avoid numerical instabilities and enlarge the domain of feasibility of the problem, the terminal constraint is commonly expressed as an inequality constraint. Therefore, the reachability

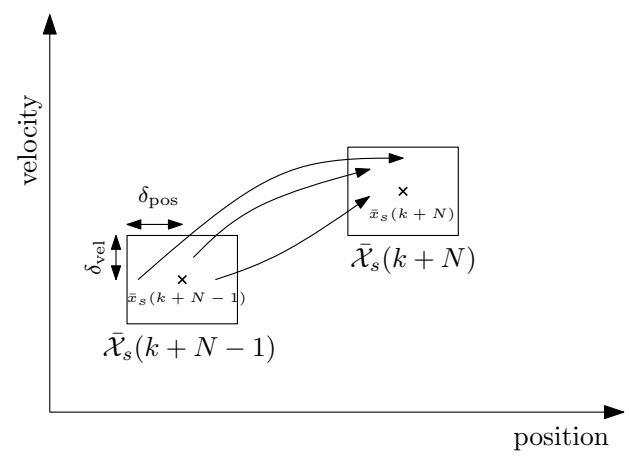

Figure 4. Terminal equality constraint

condition (11) can be formulated as

$$
\operatorname{Pre}\left(\bar{X}_{s}(k+N)\right) \cap \bar{X}_{s}(k+N-1)=\bar{X}_{s}(k+N-1)
$$

which can be more efficiently computed, and where the terminal set is defined as

$$
\bar{X}_{s}=\left\{x \in \mathbb{R}^{n}:-\left[\delta_{\mathrm{pos}} \delta_{\mathrm{vel}}\right]^{\prime} \leq x-\bar{x}_{s}(k) \leq+\left[\delta_{\mathrm{pos}} \delta_{\mathrm{vel}}\right]^{\prime}\right\}
$$

and is shown in Fig. 4 and Pre is defined as

$$
\operatorname{Pre}(X)=\left\{x \in \mathbb{R}^{n}: \exists u \in \mathcal{U} \text { s.t. } A x+B u \in X\right\}
$$

Further details on Pre and Reach sets can be found in [12]. Computation of Pre-set for a controlled system involves set projection. Here, we propose a different approach for evaluating condition (13).

Proposition 1. Given $x(k+1)=A x(k)+B u(k)$, a polytope of admissible input $\mathcal{U}$, a polytope $\mathcal{X}$ with vertices $V=\left\{v_{1}, \cdots, v_{e}\right\}$, and a polytope $X^{+}$, if for every $v_{i}, i=1, \cdots, e$, there exists $u_{i} \in \mathcal{U}$ such that $A v_{i}+B u_{i} \in \mathcal{X}^{+}$, then for every $x \in \mathcal{X}$, there exists $u \in \mathcal{U}$ such that $A x+B u \in X^{+}$

The proof of Proposition 1 is given in Appendix.

Using Proposition 1, condition (13) can be evaluated, more conservatively, without performing set projection by simply evaluating the following condition.

$$
\begin{array}{r}
\forall x_{i} \in V_{\bar{X}_{s}(k+N-1)}, \exists x_{j} \in V_{\bar{X}_{s}(k+N)} \\
\text { s.t. }\left(x_{j}-A x_{i}\right) \in B \circ \mathcal{U}
\end{array}
$$

where $V_{\bar{X}_{s}(k+N)}$ is the set of vertices of $\bar{X}_{s}(k+N), \mathcal{U}$ is the set of admissible control inputs, and $\circ$ represents the application of a 
linear mapping to a polyhedron, which results in a new polyhedron. Condition (16) verifies whether the system can be driven from all vertices of the set $\bar{X}_{s}(k+N-1)$ to at least one vertex of the set $\bar{X}_{s}(k+N)$.

3.1.2 INTER-SAMPLE TRACKING In addition to the recursive feasibility requirement, to guarantee the satisfaction of the fine stage stroke constraint, $\bar{y}_{f}=r e f-y_{s}$ needs to be within the stroke range of the fine stage. Assuming that the terminal constraint can be satisfied (for steps $k-1+N$ and $k+N$ ), as obtained from Section 3.1.1, the last piece of the coarse stage trajectory is considered to be connecting $\bar{y}_{s}(k+N-1)$ to $\bar{y}_{s}(k+N)$. This is shown in Fig. 5. It should be noted that the coarse stage is assumed to be moving linearly between two consecutive sample points. This is reasonable when the friction in (3) is small, and/or the axes experience very similar motions. The error introduced by this approximation can be computed and used to appropriately tighten the constraints. Given the motion of the coarse stage and the fine stage stroke limit $\left(d_{\max , f}\right)$, the infeasible areas for tracking can be found. As it can be seen from Fig. 5, due to the significant difference between the sampling periods of the two stage $\left(T_{f}<<T_{s}\right)$ and the fact that the target reference trajectory is generated using the fine stage maximal acceleration and velocity (which are significantly higher than the one's of coarse stage), the stroke constraint can be violated even if the target reference trajectory is within the stroke range at time steps $k+N-1$ and $k+N$. Therefore, to guarantee stroke constraint satisfaction, the inter-sample locations of the target reference trajectory have to be examined.

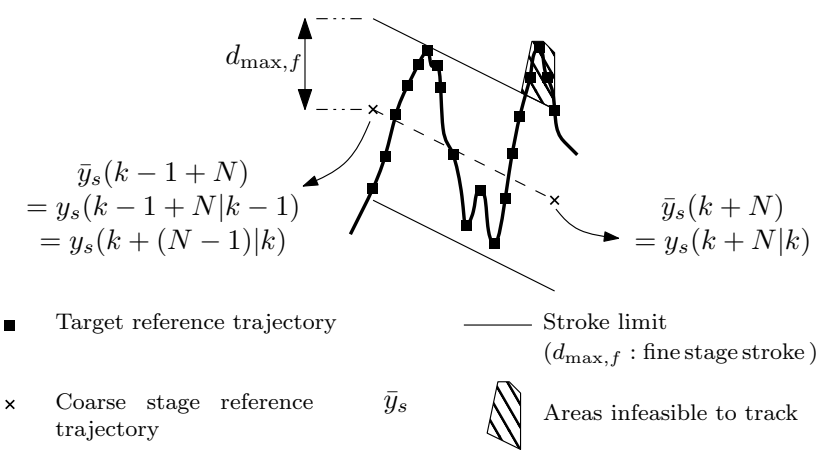

Figure 5. Tracking constraint in the original form

Using the following inequalities, the inter-sample constraints can be evaluated

$$
\left\{\begin{array}{c}
\operatorname{ref}\left((k+N-1) N_{f}+j\right)< \\
\bar{y}_{s}(k-1+N)+d_{\max , f}+\left(\frac{\bar{y}_{s}(k+N)-\bar{y}_{s}(k-1+N)}{T_{s}}\right) j T_{f} \\
\operatorname{ref}\left((k+N-1) N_{f}+j\right)> \\
\bar{y}_{s}(k-1+N)-d_{\max , f}+\left(\frac{\bar{y}_{s}(k+N)-\bar{y}_{s}(k-1+N)}{T_{s}}\right) j T_{f}
\end{array}\right.
$$

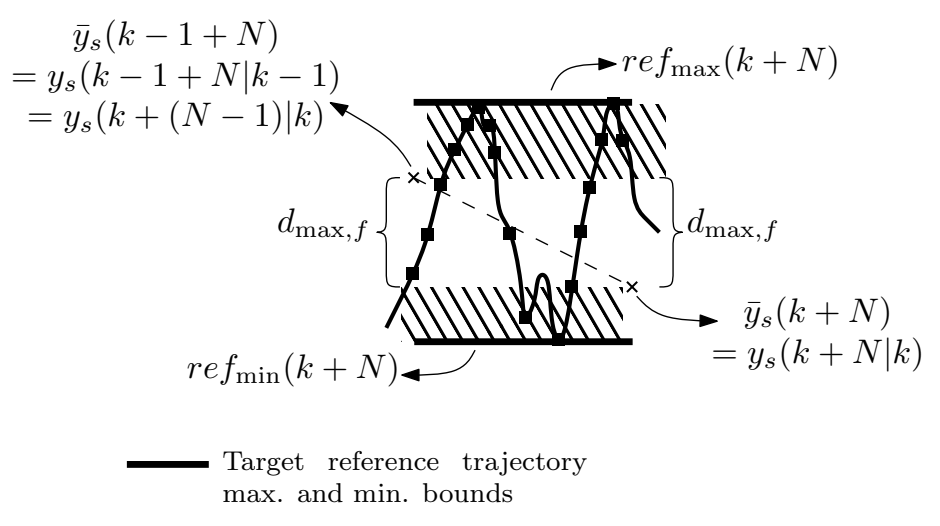

Figure 6. Modified tracking constraint

where $j=0,1, \cdots, N_{f}$. Constraints in (17) have to be evaluated at $N_{f}$ points, which requires a significant amount of computation, since $N_{f}$ is a large number. Furthermore, such constraints cannot be easily implemented in an MPC problem. To work around this issue, a more conservative but more computationally tractable approach is employed. The proposed approach is based on computing maximal and minimal values for the target reference trajectory during each sampling period of the coarse stage. The idea is depicted in Fig. 6. The maximal and minimal values are computed by

$$
\begin{array}{r}
r e f_{\max }(k+N)=\max \left\{r e f\left((k-1+N) N_{f}+j\right)\right\} \\
r e f_{\min }(k+N)=\min \left\{r e f\left((k-1+N) N_{f}+j\right)\right\} \\
j=0, \cdots, N_{f}
\end{array}
$$

Using the bounds in (18), the satisfaction of the inter-sample tracking constraints can be evaluated using the following inequalities

$$
\left\{\begin{array}{c}
\left|r e f_{\max }(k+N)-\bar{y}_{s}(k-1+N)\right| \leq d_{\max , f} \\
\left|r e f_{\min }(k+N)-\bar{y}_{s}(k-1+N)\right| \leq d_{\max , f} \\
\left|r e f_{\max }(k+N)-\bar{y}_{s}(k+N)\right| \leq d_{\max , f} \\
\left|r e f_{\min }(k+N)-\bar{y}_{s}(k+N)\right| \leq d_{\max , f}
\end{array}\right.
$$

Finally, since in Section 3.1.1 we impose terminal set constraints rather than a terminal point constraint, each inequality in (19) is replaced with two inequalities, where $\bar{y}_{s}$ is once replaced with $\bar{y}_{s}+\delta_{\text {pos }}$ and another time with $\bar{y}_{s}-\delta_{\text {pos }}$. Constraints (16) and (19) can be used to design a governor to generate feasible reference trajectories for Problem 1, as described next.

\subsection{GOVERNOR DESIGN}

To ensure sure that the MPC works properly, we need to make sure that a feasible solution to the optimal control problem (7) always exists. To guarantee this, we generate a reference 
trajectory for the coarse stage that is guaranteed to be feasible, so that it is a feasible solution of the MPC problem. As discussed in Section 3, a candidate target reference trajectory is constructed by joining sub-trajectories that are generated using the fine stage maximal velocity and acceleration limits. Therefore, any infeasibility in the reference trajectory is necessarily due to the violation of conditions (16) and/or (19). If the reference trajectory happens to be infeasible, part of it has to be generated using a reduced speed and a reduced acceleration, i.e., the target reference trajectory has to be slowed down. To regain feasibility at time step $k+N$, the reference trajectory has to be slowed down from time $(k-1+N) T_{s}$ to $(k+N) T_{s}+N_{\text {fir }} T_{f}$. The slowdown problem can be cast as the optimization problem

$$
\begin{gathered}
\max _{a_{\max }, v_{\max }} \sum_{i=(k-1+N) \frac{T_{s}}{T_{f}}}^{(k+N) \frac{T_{s}}{T_{f}}+N_{\text {fir }}}\left(\left([\operatorname{ref}(i+1)]_{1}-[\operatorname{ref}(i)]_{1}\right)^{2}\right. \\
\left.+\left([\operatorname{ref}(i+1)]_{2}-[\operatorname{ref}(i)]_{2}\right)^{2}\right) \\
\text { s.t. } \quad \text { conditions }(16) \text { and }(19) \\
\\
\\
\quad\left|a_{\max }\right| \leq a_{\max , f} \\
\\
\left|v_{\max }\right| \leq v_{\max , f} .
\end{gathered}
$$

In this work, an iterative approach is used to solve (20) and to regain feasibility. The proposed slowdown algorithm is reported in Algorithm 1. The slowdown factor $\left(\sigma_{\mathrm{sd}}\right)$ is set to 0.9 .

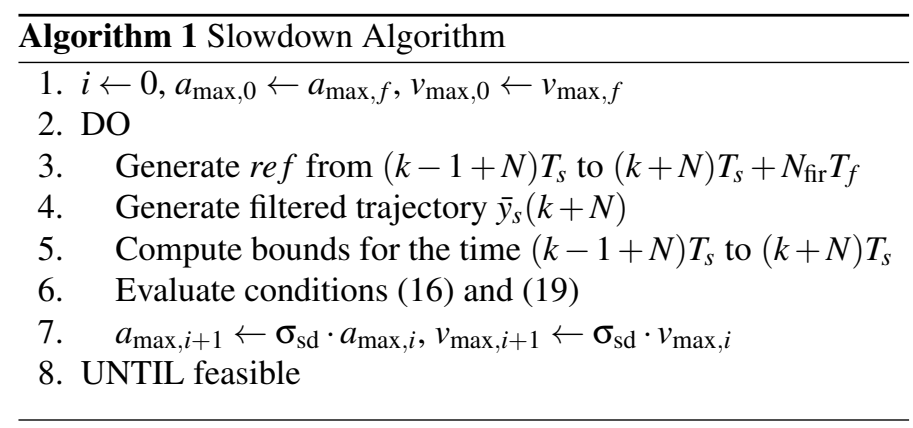

\section{MPC DESIGN FOR THE COARSE STAGE}

Next we design an MPC controller to solve Problem 2. The techniques in Section 3 provide a feasible trajectory that is used as reference for the MPC controller. The trajectory generated by MPC is optimal while the feasibility of the reference trajectory guarantees that a solution to the MPC problem always exists. Note that since there are constraints between the MPC reference and the states of the coarse stage, without applying the techniques of Section 3 the MPC problem may become infeasible and hence the machine will fail at perfectly tracking the target reference trajectory. The fact that the reference trajectory is feasible does not imply that any control system can simply follow the reference trajectory while satisfying all constraints. Therefore, as mentioned before, to achieve optimality and satisfy system constraints we use MPC to solve Problem 2 (controlling the coarse stage). A quadratic cost function of the following form is used in the MPC design,

$$
\begin{array}{r}
J(U(k))=\sum_{i=1}^{N}\left\|\bar{y}_{s}(k+i)-y_{s}(k+i)\right\|_{2}^{Q_{1}}+\left\|x_{s}(k+i)\right\|_{2}^{Q_{2}}+ \\
\left\|u_{s}(k+i-1)\right\|_{2}^{R} .
\end{array}
$$

where $U(k)$ is the sequence of control inputs and is defines as $U(k)=\left[u_{s}(k), u_{s}(k+1), \cdots, u_{s}(k+N-1)\right]^{\prime}$. For controlling the coarse stage, we consider $Q_{2}$ in the form $\left[\begin{array}{ll}0 & 0 \\ 0 & q_{2}\end{array}\right]$.

\subsection{CONSTRAINTS}

The system constraints are grouped into three categories: 1) constraints on the control $\left.u_{s}(k), 2\right)$ constraints on the state $x_{s}(k)$, and 3 ) constraints on the output $y_{s}(k)$ due to the fine stage limited stroke.

4.1.1 INPUT CONSTRAINTS As discussed in Section 2 , the control torque provided by the servomotors $u_{s}(k)$ is limited.

$$
\left|u_{s}(k+i)\right| \leq u_{\max , s}, i=0, \cdots, N-1 .
$$

4.1.2 STATE CONSTRAINTS Two types of state constraints are considered in this work. The first one enforces operational limits of the coarse stage (stroke and speed). In addition, the system trajectory has to reach the terminal set at the end of the prediction horizon $N$. As mentioned before this constraint is needed to guarantee recursive feasibility of the MPC problem [11].

$$
\begin{aligned}
\left|x_{s}(k+i)\right| \leq & {\left[\begin{array}{l}
d_{\max , s} \\
v_{\max , s}
\end{array}\right], i=1, \cdots, N } \\
& x_{S}(k+N) \in \bar{X}_{s}(k+N) .
\end{aligned}
$$

4.1.3 OUTPUT CONSTRAINTS Using the target reference trajectory maximal and minimal bounds (18), the output 
constraints enforce $\left|\bar{y}_{f}\right| \leq d_{\max , f}$ and are expressed as,

$$
\begin{aligned}
& \left|y_{s}(k+i)-r e f_{\max }(k+i)\right|<d_{\max , f} \\
& \left|y_{s}(k+i)-r e f_{\max }(k+i+1)\right|<d_{\max , f} \\
& \left|y_{s}(k+i)-r e f_{\min }(k+i)\right|<d_{\max , f} \\
& \left|y_{s}(k+i)-r e f_{\min }(k+i+1)\right|<d_{\max , f}, \\
& i=1, \cdots, N .
\end{aligned}
$$

\subsection{MPC PROBLEM}

The MPC problem at every sampling time solves the following finite horizon optimal control problem.

$$
\begin{array}{ll}
\min _{U(k)} & \sum_{i=1}^{N}\left\|\bar{y}_{s}(k+i)-y_{s}(k+i)\right\|_{2}^{Q_{1}}+\left\|x_{s}(k+i)\right\|_{2}^{Q_{2}} \\
& +\left\|u_{s}(k+i-1)\right\|_{2}^{R} \\
\text { s.t. } & x_{s}(k+1)=A x_{s}(k)+B u_{s}(k) \\
& y_{s}(k)=C x_{s}(k) \\
& \left|u_{s}(k+i)\right| \leq u_{\max , s}, i=0, \cdots, N-1 \\
& \left|x_{s}(k+i)\right| \leq\left[\begin{array}{l}
d_{\max , s} \\
v_{\max , s}
\end{array}\right] \\
& \left|y_{s}(k+i)-r e f_{\max }(k+i)\right|<d_{\max , f} \\
& \left|y_{s}(k+i)-r e f_{\max }(k+i+1)\right|<d_{\max , f} \\
& \left|y_{s}(k+i)-r e f_{\min }(k+i)\right|<d_{\max , f} \\
& \left|y_{s}(k+i)-r e f_{\min }(k+i+1)\right|<d_{\max , f}, \\
& x_{s}(k+N) \in \bar{X}_{s}(k+N) . \quad i=1, \cdots, N
\end{array}
$$

At time step $k$, the optimal control problem (25) is solved to find $U(k)$. The first element of the optimal control sequence, $U(k)$, is then applied to the coarse stage. The system evolves according to its dynamics until the next sampling period when the system states are re-measured, and the optimization problem is solved again.

In this paper, we solve Problem (25) using the PQP-MPC algorithm proposed in [13]. To this end, we formulate (25) as parametric quadratic programing

$$
\begin{aligned}
\min _{U}(k) & \frac{1}{2} U^{\prime} Q_{p} U+\theta^{\prime} C_{p}^{\prime} U+\frac{1}{2} \theta^{\prime} \Omega_{p} \theta \\
\text { s.t. } & G_{p} U \leq S_{p} \theta+W_{p} .
\end{aligned}
$$

where $U=U(k)$, the parameter vector $\theta$ contains the current state $(x(k))$, the current references for the coarse stage $\left(\bar{y}_{s}(k+i), i=1, \cdots, N\right)$, and the current reference bounds $\left(r e f_{\max }(k+i), r e f_{\min }(k+i), i=1, \cdots, N\right)$ for Problem (25).
The matrices of the primal problem $Q_{p} \in \mathbb{R}^{n_{u} \times n_{u}}, n_{u}=N_{u} m$, $Q_{p}>0, G_{p} \in \mathbb{R}^{n_{c} \times n_{u}}$ ( $n_{c}$ is the total number of constraints), $C_{p} \in \mathbb{R}^{n_{u} \times n_{\theta}}\left(n_{\theta}=n_{x}+3 N\right)$, and $\Omega_{p}$ are pre-computed as explained, for instance, in [14]. In the PQP-MPC algorithm, the parametric dual problem of (26) is formulated as

$$
\begin{array}{ll}
\min _{Y} & \frac{1}{2} Y^{\prime} Q_{d} Y+\left(\theta^{\prime} S_{d}^{\prime}+W_{d}^{\prime}\right) Y+\frac{1}{2} \theta^{\prime} \Omega_{d} \theta \\
\text { s.t. } & Y \geq 0
\end{array}
$$

where $Q_{d}=G_{p} Q_{P}^{-1} G_{p}^{\prime}, S_{d}=\left(G_{p} Q_{p}^{-1} C_{p}+S_{p}\right), W_{d}=W_{p}, \Omega_{d}=$ $C_{p}^{\prime} Q_{p}^{-1} C_{p}-\Omega_{p}$. Then starting from an arbitrary $Y>0$, the solution of (27) is computed by iterating over the following multiplicative update law

$$
\left[Y_{(h+1)}\right]_{i}=\frac{\left[\left(Q_{d}^{-}+\phi\right) Y_{(h)}+\left(S_{d} \theta+W_{d}\right)^{-}\right]_{i}}{\left[\left(Q_{d}^{+}+\phi\right) Y_{(h)}+\left(S_{D} \theta+W_{d}\right)^{+}\right]_{i}}\left[Y_{(h)}\right]_{i},
$$

until a termination condition based on the values of primal and dual cost and constraints satisfaction is reached. Further details on the algorithm and the proof of convergence are available in [13]. The advantages of using PQP-MPC algorithm (28) to solve (26) is in the algorithm simple code, small memory footprint, and the fact that it is very fast for finding the solution of these types of problem, making it ideal for real-time implementation.

\section{RESULTS}

In this paper, a linear second-order system (3) is used to represent the coarse stage dynamics. A generic 2-dimensional pattern is used to perform numerical simulation and evaluate the performance of the proposed method. The sampling period for the coarse stage, $T_{s}$, is $0.025 \mathrm{~s}$ and the prediction horizon, $N$, is set to 40. The terminal constraint set is constructed by setting $\delta_{\text {pos }}$ to $0.003 \mathrm{~m}$ and setting $\delta_{\text {vel }}$ to $0.05 \mathrm{~m} / \mathrm{s}$. The dual-stage machine can inspect the whole pattern in 21.85 seconds. The inspection time is independent of the weighting matrices of the MPC problem and the prediction horizon as it is driven by the reference trajectory which is generated to satisfy the constraints. However, the size of the terminal set, which is set by $\delta_{\text {pos }}$ and $\delta_{\text {vel }}$ can affect the inspection time. Table 1 shows the dependence of the inspection time on the size of the terminal set. It should also be noted that the terminal set constraint (25j) is always active. Therefore, the size of the terminal set can affect the computation time. For the terminal set used here $\left(\delta_{\mathrm{pos}}=0.003 \mathrm{~m}\right.$ and $\left.\delta_{\mathrm{vel}}=0.05 \mathrm{~m} / \mathrm{s}\right)$, the average computation time at each time step, using a single core of a $2.6 \mathrm{GHz}$ Intel core i7 processor, is $0.0047 \mathrm{~s}$ which is considerably shorter than the sampling period $\left(T_{s}=0.025 \mathrm{~s}\right)$. 


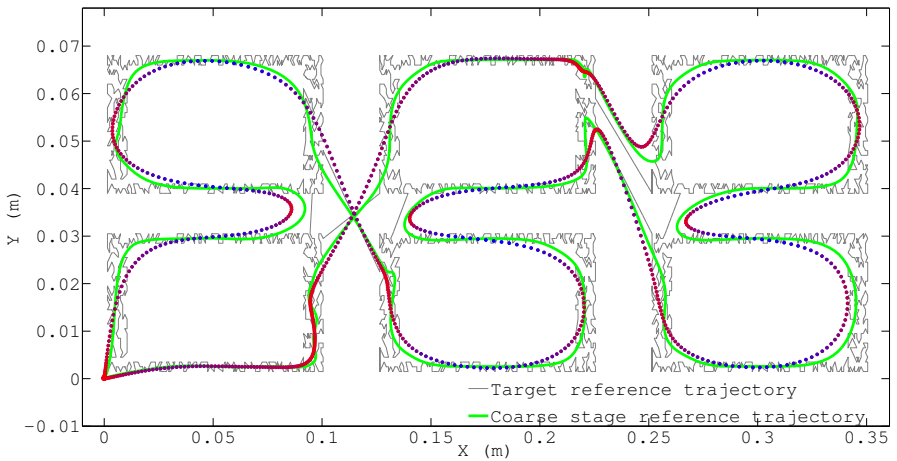

Figure 7. $\mathrm{X}-\mathrm{Y}$ motion pattern of the target reference trajectory and the coarse stage

Table 1. Inspection time dependence on the size of the terminal constraint set

\begin{tabular}{|c|c|c|c|c|c|}
\hline$\delta_{\text {vel }} \delta_{\text {pos }}$ & 0.001 & 0.002 & 0.003 & 0.004 & 0.005 \\
\hline 0.025 & 15.65 & 18.6 & 20.025 & 23.65 & 26.025 \\
\hline 0.05 & 15.325 & 17.95 & 21.85 & 24.85 & 26.025 \\
\hline 0.075 & 24.775 & 24.85 & 27.375 & 28.5 & 32.975 \\
\hline
\end{tabular}

Figure 7 shows the inspected pattern and the generated feasible reference trajectory for the coarse stage. The dotted line represents the coarse stage actual position. The position line is color coded using the coarse stage speed at each point where blue represents the maximum speed and red represents zero speed. The maximum speed attained by the coarse stage is $0.1449 \mathrm{~m} / \mathrm{s}$ which is considerably lower than its maximal speed of $0.95 \mathrm{~m} / \mathrm{s}$. This is due to the density of the target points and the restrictions imposed by the fine stage stroke. Note that maximum speed is attained while traveling along the $\mathrm{X}$-direction. This is due to the fact the cells in the inspected pattern are rectangular with the horizontal side three times longer than the vertical side, hence the coarse stage accelerates more in the horizontal direction. Figures 8 and 9 show the coarse stage trajectory along the $\mathrm{X}$ and $\mathrm{Y}$ axes. As it can be seen from Figures 7 to 9, MPC steers the coarse stage in a smooth way, while keeping the target reference trajectory within the stroke range of the fine stage. Figures 10 and 11 show the stroke, velocity, and torque constraints for the coarse stage, which are all satisfied. We realize that, rather than the coarse stage constraints, the fine stage stroke constraint is the limiting factor for the dual-stage positioning system, which motivates the approach developed here.

\section{CONCLUSIONS}

In this paper, we have described an architecture for receding horizon control of dual-stage positioning machines that guaran-

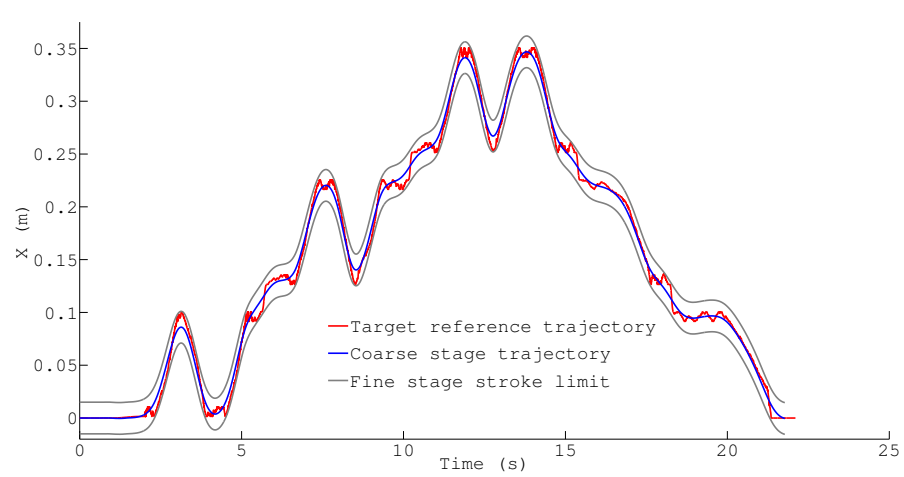

Figure 8. Reference and coarse stage trajectory (X-axis)

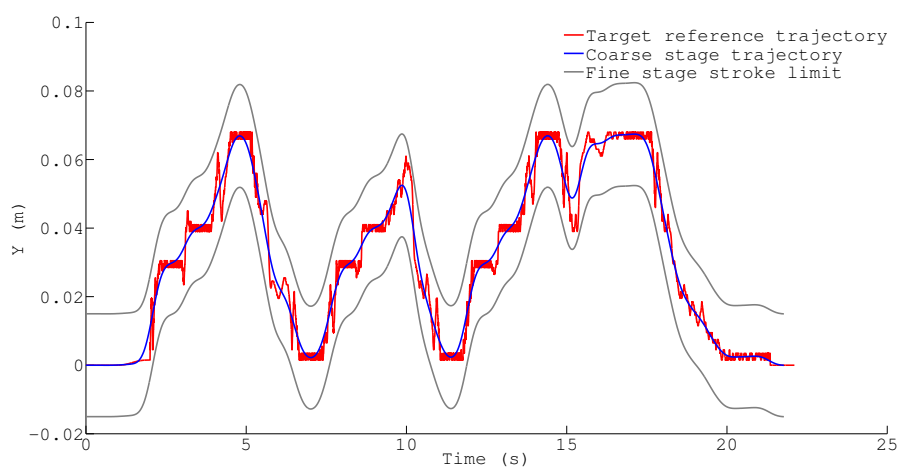

Figure 9. Reference and coarse stage trajectory (Y-axis)
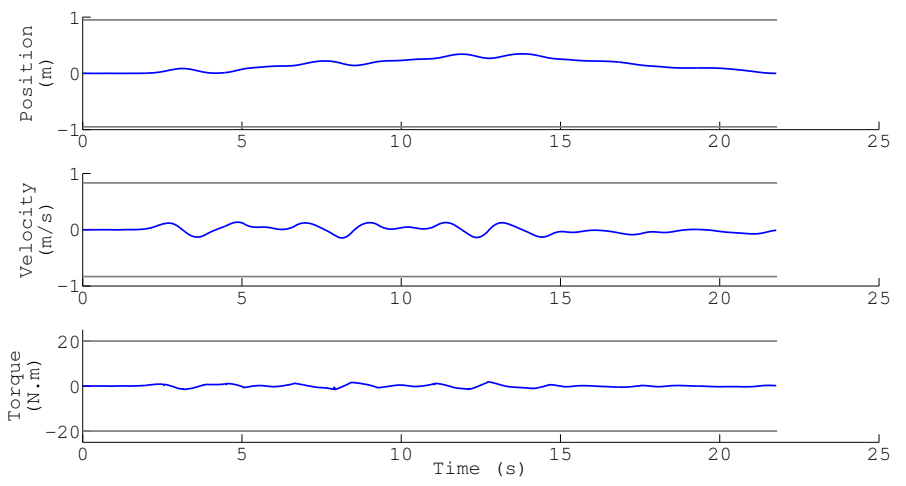

Figure 10. Coarse stage position, velocity, and torque time history ( $X$ axis)

tees constraints satisfaction. We first extend the target reference trajectory (and the coarse stage reference trajectory) such that the fine stage constraints are satisfied and verifying that a terminal constraint and an inter-sample tracking constraints are satisfied. The reference trajectory for the coarse stage is then used as reference in an MPC controller that obtains the optimal trajectory for the coarse stage while guaranteeing that all the constraints are satisfied. Since the reference trajectory is feasible and terminal 

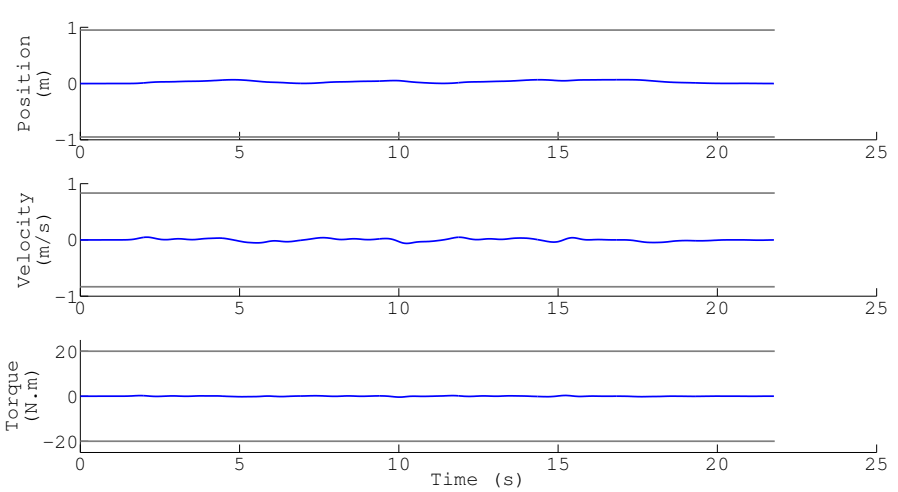

Figure 11. Coarse stage position, velocity, and torque time history (Yaxis)

constraint is included in the MPC formulation, recursive feasibility of the MPC problem is guaranteed. Furthermore, the introduction of maximal and minimal bounds for the target reference trajectory has allowed us to efficiently implement inter-sample tracking constraints in MPC problem formulation, which results in the satisfaction of the fine stage stroke constraint. We have shown the results of the proposed techniques on a generic pattern. The obtained results have shown that all system constraints are satisfied. We have realized from the results that the fine stage stroke constraint is often the limiting factor in dual-stage positioning systems further motivating the approach developed here.

\section{REFERENCES}

[1] Salcudean, S., and An, C., 1989. "On the control of redundant coarse-fine manipulators". In IEEE International Conference on Robotics and Automation.

[2] Staroselsky, S., and Stelson, K. A., 1988. "Two-stage actuation for improved accuracy of contouring”. In American Control Confernce.

[3] Kwon, S., Chung, W. K., and Youm, Y., 2001. "On the coarse/fine dual-stage manipulators with robust perturbation compensator". In IEEE International Conference on Robotics and Automation.

[4] Kwon, S., and Cheong, J., 2006. "Robust minimum-time control with coarse/fine dual-stage mechanism". Journal of Mechanical Science and Technology, 20(11), pp. 18341847.

[5] Schroeck, S. J., Messner, W. C., and McNab, R. J., 2001. "On compensator design for linear time-invarinat dual-input single-output systems". IEEE Transactions on Mechatronics, 6(1), March, pp. 50-57.

[6] Ryoo, J. R., Doh, T.-Y., and Chung, M. J., 2005. “Compensator design for a dual-stage actuator in the track-following servo system of optical disk drives". IEEE Transactions on Consumer Electronics, 51(2), May, pp. 471-477.
[7] Boettcher, U., Callafon, R. A. D., and Talke, F. E., 2010. "Modeling and control of a dual stage actuator hard disk drive”. Journal of Advanced Mechanical Design, Systems, and Manufacturing, 4(1), pp. 107-118.

[8] Michellod, Y., Mullhaupt, P., and Gillet, D., 2006. "Strategy for the control of a dual-stage nano-positioning system with a single metrology". In IEEE Conference on Robotics, Automation and Mechatronics.

[9] Lam, D., Manzie, C., and Good, M. C., 2013. "Model predictive contouring control for biaxial systems". IEEE Transactions on Control Systems Technology, 21(2), March, pp. 552-559.

[10] Hara, S., Hara, T., Yi, L., and Tomizuka, M., 2000. "Novel reference signal generation for two-degree-of-freedom contcontrol for hard disk drives". IEEE Transactions on Mechatronics, 5(1), March, pp. 73-78.

[11] Mayne, D. Q., Rawlings, J. B., Rao, C. V., and Scokaert, P. O. M., 2000. "Consrtained model predictive control: Stability and optimality”. Automatica, 36(6), June, pp. 789814.

[12] Kerrigan, E. C., 2000. "Robust constraints satisfaction: Invariant sets and predictive control". PhD thesis, Dep. of Engineering, University of Cambridge, Cambridge, UK.

[13] Di Cairano, S., Brand, M., and Bortoff, S. A., 2013. "Projection-free parallel quadratic programming for linear model predictive control". International Journal of Control, 86(8), pp. 1367-1385.

[14] Maciejowski, J., 2002. Predictive control with constraints. Prentice Hall.

\section{APPENDIX: PROOF OF PROPOSITION 1}

If every vertex of set $X$ can be feasibly driven to at least one point $x_{i}^{+} \in \mathcal{X}^{+}$, then any point $x \in \mathcal{X}$ can be feasibly driven at least a point in $\mathcal{X}^{+}$, that is,

$$
\forall v_{i} \in V_{X}, \exists x_{i}^{+} \in \mathcal{X}^{+}, \exists u_{i} \in \mathcal{U} \text { s.t. } x_{i}^{+}=A v_{i}+B u_{i} .
$$

By convexity, any point $x \in \mathcal{X}$ can be written as

$$
x=\sum_{i=1}^{\ell} \lambda_{i} x_{i} \text { s.t. } \sum_{i=1}^{\ell} \lambda_{i}=1, \lambda_{i} \geq 0,
$$

where $\ell$ is the number of vertices of the set $X$. By taking summation over (29),

$$
\sum_{i=1}^{\ell} \lambda_{i}\left(x_{i}^{+}=A x_{i}+B u_{i}\right) \Rightarrow \underbrace{\sum_{i=1}^{\ell} \lambda_{i} x_{i}^{+}}_{x^{+}}=A x+B \sum_{i=1}^{\ell} \lambda_{i} u_{i} .
$$

By convexity $\sum_{i=1}^{\ell} \lambda_{i} u_{i} \in \mathcal{U}$ and $x^{+} \in \mathcal{X}^{+}$which finishes the proof. 